\title{
When cooperation goes wrong: Brain and behavioral correlates of ineffective joint strategies in dyads.
}

\section{Michela Balconi, Laura Gatti \& Maria Elide Vanutelli}

To cite this article: Michela Balconi, Laura Gatti \& Maria Elide Vanutelli (2017): When cooperation goes wrong: Brain and behavioral correlates of ineffective joint strategies in dyads., International Journal of Neuroscience, DOI: 10.1080/00207454.2017.1379519

To link to this article: http://dx.doi.org/10.1080/00207454.2017.1379519

Accepted author version posted online: 15

Sep 2017.

Submit your article to this journal $\llbracket$

Q View related articles $\widetilde{2}$

View Crossmark data $₫$ 
Publisher: Taylor \& Francis

Journal: International Journal of Neuroscience

DOI: https://doi.org/10.1080/00207454.2017.1379519

When cooperation goes wrong: Brain and behavioral correlates of ineffective joint strategies in dyads.

Michela Balconi ${ }^{1,2}$, Laura Gatti $^{2}$, \& Maria Elide Vanutelli ${ }^{1}$

${ }^{1}$ Research Unit in Affective and Social Neuroscience, Catholic University of Milan, 20123, Milan, Italy

${ }^{2}$ Department of Psychology, Catholic University of Milan, 20123, Milan, Italy

Running Head:

When cooperation goes wrong

* Corresponding author

$\underline{\text { Michela Balconi }}$

Department of Psychology, Catholic University of the Sacred Heart, Milan

Largo Gemelli, 1, 20123, Milan, Italy

Tel: +39-2-72342586; fax: $+30-2-72342233$

E-mail address: $\underline{\text { michela.balconi@ unicatt.it }}$

ORCID ID: 0000-0002-8634-1951 


\section{$\underline{\text { Maria Elide Vanutelli }}$}

Department of Psychology, Catholic University of the Sacred Heart, Milan

Largo Gemelli, 1, 20123, Milan, Italy

Tel: +39-2-72345929

E-mail address: mariaelide.vanutelli@unicatt.it

ORCID ID: 0000-0001-9349-1707

\section{$\underline{\text { Laura Gatti }}$}

Department of Psychology, Catholic University of the Sacred Heart, Milan

Largo Gemelli, 1, 20123, Milan, Italy

Tel: $+39-2-72345929$

E-mail address: laura.gatti02@icatt.it

Michela Balconi, PhD, is Professor of 'Neuropsychology and Cognitive Neuroscience', 'Neuropsychology of Communication', and 'Neuroscience of Well-being in the Lifespan' at the Faculty of Psychology of the Catholic University of the Sacred Heart, Milan and Brescia. She leads the Research Unit in Affective and Social Neuroscience and manages the research activities of the Laboratory for Cognitive Psychology and of many assistants and collaborators. Her research interests mainly concern Cognitive Neuropsychology and Psychophysiology. In agreement with the relevance of a proper integration between the body 
and the mind, she has studied and introduced new methods to analyze and explore the relation between affective, communication and cognitive processes and physiological markers - e.g. the application of fNIRS (functional Near-Infrared Spectroscopy) technique and EEG frequency bands analysis to the investigation of social-affective processes and consciousness correlates, and the use of non-invasive brain stimulation (TMS, Transcranial Magnetic Stimulation, and tES, transcranial Electric Stimulation) in clinical and experimental contexts.

Maria Elide Vanutelli took her PhD in Psychology at the Catholic University of the Sacred Heart, Milan, with a thesis entitled 'Sharing emotions in social life: New perspectives in interactive neuroscience'. Her main research interests concern social neuroscience with a specific focus on affective and empathic mechanisms in interpersonal relationships.

Laura Gatti graduated at the Catholic University of the Sacred Heart, Brescia, and she is currently spending her apprenticeship on the topics of cooperation, with a specific interest for gender differences during joint actions. 


\begin{abstract}
Human life is connoted by sophisticated interactions that involve not only single individuals, but larger social groups composed by members interacting each other. Cooperation secures a benefit to all the people engaged as well as important behaviors like helping, sharing, and acting prosocially. But what happens when the joint actions are not effective? In the present study we asked 24 participants paired in 12 dyads to cooperate during an attentional task in a way to synchronize their responses and obtain better outcomes. In addition we tested interbrain and cognitive strategy similarities between subjects. Then, we frustrated their strategies by providing false feedbacks signaling the incapacity to create a synergy, which was reinforced by a general negative evaluation halfway through the task. The effects of the feedback in modulating subjects' behavioral performance and brain responsiveness were explored by means of functional near-infrared spectroscopy (fNIRS). Results showed a worsen performance after the negative feedback in the form of longer reaction times (RTs) and a specific pattern of brain activation involving the dorsolateral prefrontal cortex (DLPFC) and the superior frontal gyrus (SFG). The DLPFC showed increased O2Hb (oxy-hemoglobin) level after the feedback, compatible with the need for higher cognitive effort. In addition, fNIRS measures revealed a decreased inter-brain synchronicity in post-feedback condition for the dyad. Also, the representation of negative emotions in response to failing interactions was signaled by a right-lateralized effect. Results were interpreted at light of available knowledge on perceived self-efficacy and the implementation of common goals and strategies.
\end{abstract}

Keywords: cooperation, self-efficacy, fNIRS, performance, hyperscanning 


\section{Introduction}

The term cooperation is usually used to describe collaborative actions implying two or more individuals and the production of common behavioral effects. This kind of behavior is planned, realized and directed towards a specific aim or the completion of actions which implies a common interest, and generally secures a benefit to all the people involved. This representation can comprise actors operating in different domains like helping, reciprocally sharing, or acting prosocially.

The construction of these joint actions involves higher cognitive and emotional mechanisms. As a possible fulcrum of such processes the capacity to perceive and infer others' affective states could be relevant, from more basic resonance and mirroring abilities, towards the development of complex social sharing based on joint attention and synchronization [1-4]. In other words, the higher the capacities to sync and infer the consequences of different actions on others life are, the more frequent and complex the attempts to act cooperatively will be [5].

In parallel to synchronized joint actions, cooperative performances during an interpersonal task essentially imply a process of social comparison. Some previous studies explored the effect of cooperation on self-perception, perceived efficacy in social interactions, and social cognition within the social hierarchy. Such studies showed that a cooperative condition may reinforce the sense of being part of a group and it may increase the sense of self-efficacy, the general social well-being, and the perception of higher social positions [6-10]. It was also shown that cooperative strategies reinforce interpersonal cohesion, whereas in some cases the outcomes are less effective than in competitive conditions [9].

Considering the neuroscientific perspective, recent research examined the structure and function of brain areas associated with social perception and efficacy. Results suggested the 
contribution of prefrontal neural mechanism in response to cooperative tasks $[2,3,8,11]$. Indeed, it was observed that neural circuits linking limbic regions, the prefrontal cortex (PFC), and striatal structures may support the emotional, cognitive, and behavioral components of social interactions during cooperation [12]. Specifically, it was found that the dorsal (DLPFC) and ventral (VLPFC) portions of the lateral PFC are generally engaged during social status inferences $[6,13,14]$. The activation of DLPFC and VLPFC during social interactions that involve status perception probably reflects the recruitment of top-down control mechanisms over specific emotional responses to social contexts, in a way to regulate an appropriate response [15]. In fact, it should be noted that these brain regions are typically associated with the regulation of socio-emotional responses and behavioral inhibition.

In addition, some recent studies on brain coherence and connectivity found a positive relation between performance and task-related coherence, indicating that better task performance coincides with greater task-related inter-brain coherence [3]. Strong inter-brain neural synchrony was specifically observed in the posterior region of the right middle and superior frontal gyrus during cooperative exchange, suggesting that this area is involved in goal-oriented social interaction such as complex dynamic and social decision-making [16]. Inter-brain synchronicity was also observed in the dorsomedial prefrontal cortex (dmPFC) during cooperative interaction only. These additional findings suggest that this area may be particularly engaged when theory-of-mind (ToM) is required for cooperative social interaction.

However, as noted in many previous studies, an important construct that is able to mediate the brain responsiveness is the perception of successful vs ineffective inter-action. In fact, this feedback can be considered a powerful marker that can reciprocally reinforce the behavior toward a common direction, and a relevant tool to train the brain to work synergically. Previous studies explored the effect of positive outcomes on self-perception [13,17], on 
performance $[6,13,17,18]$ and brain responsiveness for cooperative or competitive tasks in respect to perceivable interpersonal feedbacks [17-19].

It was found that a positive feedback reinforces an efficient performance, activates the PFC and in many cases elicits a left-lateralized effect. Also, brain-to-brain coupling was considered at this regard, showing that a good self-representation for the positive feedback related to joint-performance reinforces the cognitive synergy, the brain effectiveness and neural synchronization [3].

At this regard, the hyperscanning approach introduces an innovative perspective to explain the joint strategy of brain-to-brain coupling [20]. However, compared to previous research, two relevant aspects were underestimated and, in our opinion, they deserve to be considered to evaluate the cooperative action effects on brain activity: the presence of a real dynamic interaction where the co-partners are actively implicated in the cooperative exchange; the active feedback furnished by an external context to represent objectively the effectiveness of the joint actions. Thus, the present study aimed at moving towards a two-person neuroscience by investigating the hemodynamic correlates of between-brain connectivity during a joint task in presence of an external feedback related to the performance.

In addition, at present no specific study directly deeply explored the influence of a negative feedback on performance and brain responsiveness. That is, when we perceive to fail based on our cooperative joint-action what kind of response do our brain and our behavior produce?

Different possible ways are suggested when an unsuccessful cooperation is selfrepresented. Firstly, a competitive behavior may be adopted, where a mental effort is required to simulate a sort of 'dysfunctional' interaction and a consequent 'disengaged' relation, since, in absence of a proficient cooperation, the synergic plan is disrupted. Indeed, it was proposed that mentalizing demands may differ in some aspects as a function of intentionality, efficacy 
and outcomes. For example, depending on the interaction modalities (positive or negative cooperation), individuals may either facilitate or hinder others' goal achievement and selfrepresent themselves as more or less proficient in relation with others. Other research demonstrated that one's own actions are facilitated when the other's actions are at the disposal of the self and are efficient [21,22]. In contrast, in the case of competition or ineffective interactions, the interlocutors' behavior is less predictable than in the case of cooperation, in which there is a planned expectation. At this regard, Gallagher and Frith [23] suggested that we need to determine an agent's mental state that is decoupled from reality, and to handle simultaneously these two views on the interactions [24]. These mechanisms rely on executive functions and, specifically, on the selection of salient knowledge or response to achieve an internally represented goal $[25,26]$. Thus, the strong increase in the prefrontal cortex activity mainly the medial prefrontal cortex - observed during competition or in the case of a failure may in part mirror higher executive processing demands [27]. Specifically, it was shown that the processing load associated with the competitive condition resulted in heightened cortical activity across all examined brain regions. As such, this condition imposed an increase in the cognitive load. Similarly, an unsuccessful strategy, although in a cooperative context, may require an increased demand of cognitive resources to update and modify the joint-action.

An alternative hypothesis may suggest the general effect of more negative emotions in a failure condition, where subjects may develop more negative and withdrawal emotions toward their own partner due to the inefficacy of the joint-action. In this case, based on the valence model of emotions, a more lateralized responsiveness should suggest a right prefrontal unbalance [28]. A further hypothesis deals with the necessity of a sort of reparative strategy to compensate the reciprocal inefficacy and to try to rich a more proficient common strategy. This second option may be represented as a sort of a resume in order to strengthen the common goals and obtain a better result. In this second case we can assume a sort of renewed 
cooperation to compensate a previous failure. This should involve some more frontal areas related to prosocial support and emotional empathic response, as reported in some studies $[29,28,30]$.

Therefore, in the present study the cortical response to this particular condition was explored. Considering its fast temporal evolution, social interaction contexts should preferably be examined by means of imaging methods that offer good resolution in both temporal and spatial domains and then allow for measuring event-related hemodynamic responses, such as functional Near-Infrared Spectroscopy (fNIRS) [31]. Importantly, NIRS is also able to provide an ecological setting in which subjects can move naturally and without constraints [32].

Therefore we planned a specific paradigm which monitored the feedback (of failure) just before (four time intervals) and just after (four time intervals) the negative feedback receipt. It was done to explore the development of the strategy and the feedback effect on the brain activity.

Based on previous hypotheses the post feedback condition (artificially inefficacious performance) could show one of these scenarios: as found in previous research on competition, a specific increased prefrontal activity is attended in order to manage an unexpected and more complex situation (failure), since subjects realized they were not efficient in synchronizing their actions; in contrast, the implication of different and selective areas of the PFC, with a specific lateralization effect, is attended since it is related to a social situation perceived as emotionally negative and uncertain from a relational point of view. Finally we expected to find significant changes in inter-brain activity after post-feedback conditions and in relationship with the negative feedback, with decreased joined-strategy. 


\section{Materials and methods}

\section{Participants}

Twenty-four undergraduate students $(\mathrm{M}=22.73, \mathrm{SD}=2.11$; male $=11)$ took part in the experiment. The participants were all right-handed and presented normal or corrected-tonormal visual acuity. They all gave informed written consent to participate in the study. Exclusion criteria were history of psychopathology (Beck Depression Inventory, BDI-H, [33]) for the subjects and immediate family. Also, State-Trait-Anxiety-Inventory (STAI, [34]) was submitted after the experimental session. Based on a clinical screening, no neurological or psychiatric pathologies were observed. No payment was provided for subjects' participation. Finally, the research was conducted in accordance with the Declaration of Helsinki and it was approved by the local ethics committee of the Department of Psychology, Catholic University of Milan.

Procedure

Subjects were comfortably seated in a moderately darkened room with a monitor screen placed approximately $60 \mathrm{~cm}$ in front of their eyes. The dyads were seated side-by-side, but separated by a black screen to prevent visual contact. They performed a simple task for sustained selective attention (it was a modified version of Balconi and Vanutelli [17]). Subjects were told that some cognitive attentional indices would have been used to evaluate the subjective skills and, to reinforce their motivation, that these measures were usually applied as a screening to test future professional career success and teamwork capabilities. In addition, the cooperative nature of the task was stressed. Indeed subjects were told that their scoring was based on the capacity to synchronize their responses, in term of both accuracy (number of correct responses: hits) and response times (RTs), with a second interlocutor (I). 
Each trial was composed by three stimuli, after which subjects received a feedback signaled by two up-arrows (high cooperation score); a dash (mean performance); or two down-arrows (low cooperation score). The feedback was presented on the screen for 5000 msec. After this feedback, an inter-trial interval (ITI) occurred and lasted for other $5000 \mathrm{msec}$. The modified version of the task was composed by two sessions: the first which did not include a specific feedback to performance (4 blocks before the feedback, 100 trials); the second which was influenced by a specific negative feedback referred to the performance (4 blocks after the feedback, 100 trials) (Fig. 1). In fact, halfway through the task, participants received a more general evaluation about their cooperative performance: actually both feedbacks and the evaluation were fixed a priori, and subjects were told during the general evaluation they had a bad cooperation (synchronicity) score with $26 \%$ in terms of speed synchrony, and $31 \%$ in terms of accuracy synchrony. They were also encouraged to change and improve their performance level during the second part of the experiment. Across the task, after the initial mean performance, subjects were constantly informed about their cooperation every three-trials by presenting the down-arrows in $70 \%$ of cases, while the dash or the up-arrows appeared in $30 \%$ of cases.

Based on a post-experiment questionnaire, participants were strongly engaged in the hierarchical context (92\% told to be strongly engaged). The subjects were also required to self-report their degree of trust of the exact feedback of the performance, which showed high trust (96\%) and the relevance of the task for the social status (94\%). Finally, negative perceived emotional condition (mainly related to negative feedback) was underlined by the participants during the task. 


\section{Performance scoring}

The reaction times (RTs, msec) were recorded from the stimulus onset, and the error rates (ERs) were calculated as the total number of incorrect detections out of the total trial, for each category. Therefore higher values represented increased incorrect responses.

\section{fNIRS}

fNIRS recordings were conducted with NIRScout System (NIRx Medical Technologies, LLC. Los Angeles, California) using an 8-channel array of optodes (4 light sources/emitters and 4 detectors) covering the prefrontal area. Emitters were placed on positions (FC3-FC4 and F1-F2) while detectors were placed on FC1-FC2 and F3-F4) (fig 2). Emitter-detector distance was kept at $30 \mathrm{~mm}$ for contiguous optodes and near-infrared light of two wavelengths (760 and $850 \mathrm{~nm}$ ) were used. NIRS optodes were placed on the subject's head using a NIRS-EEG compatible cup according to the international 10/5 system. Resulting channels were as follows: Ch 1(FC3-F3) and Ch 3 (FC4-F4) correspond to the left and right (respectively) DLPFC (Brodmann Area 9). Ch 2 (FC3-FC1) and Ch 4 (FC4-FC2) correspond to the left and right (respectively) Premotor Cortex (PMC, Brodmann Area 6). Ch 5 (F1-F3) and Ch 7 (F2-F4) correspond to the left and right (respectively) Frontal Eye Fields (FEF, Brodmann Area 8). $\mathrm{Ch} 6(\mathrm{~F} 1-\mathrm{FC} 1)$ and $\mathrm{Ch} 8(\mathrm{~F} 2-\mathrm{FC} 2)$ correspond to the left and right (respectively) Superior Frontal Gyrus (SFG, Brodmann Area 6) [35].

The changes in the concentration of oxygenated $(\mathrm{O} 2 \mathrm{Hb})$ and deoxygenated hemoglobin $(\mathrm{HHb})$ were recorded with NIRStar Acquisition Software from a $120 \mathrm{~s}$ resting period. Signals obtained from the 8 NIRS channels were obtained with a sampling rate of $6.25 \mathrm{~Hz}$ and analyzed and transformed in values for the changes in the concentration of oxy and deoxygenated hemoglobin, for each channel, scaled in mmol*mm. Oxy and deoxy-Hb 
changes were computed by using the optical density changes of 760- and 850-nm lights in accordance with the modified Beer-Lambert law. The raw data from each channel were digitally band-pass filtered at $0.01-0.3 \mathrm{~Hz}$. Then, the mean concentration of each channel was calculated by averaging data across the trials, from the trial onset for the following $5 \mathrm{~s}$. According to the mean concentrations in the time series, the effect size in every condition was calculated for each channel and subject as the difference of the means of the baseline and trial divided by the standard deviation (sd) of the baseline: $\mathrm{d}=(\mathrm{m} 1-\mathrm{m} 2) / \mathrm{s}$ (Cohen's d value) [36]. $\mathrm{M} 1$ and $\mathrm{m} 2$ are the mean concentration values during the baseline and trial, respectively, and $\mathrm{s}$ the SD of the baseline. In this case, the baseline was calculated considering the $5 \mathrm{~s}$ immediately before the trial. Then, the effect sizes obtained from the 8 channels were averaged in order to increase the signal-to-noise ratio. Although NIRS raw data were originally relative values and could not be directly averaged across subjects or channels, effect sizes normalized data could be averaged regardless of the unit [37-39]. In fact, the effect size is not affected by differential pathlength factor (DPF) [37].

\section{Results}

Four sets of analyses were performed with respect to behavioral (ERs; RTs) and neurophysiological (fNIRS: $\mathrm{O} 2 \mathrm{Hb}$ measures) measures. A preliminary repeated measure ANOVA with independent factor Condition (Cond: pre vs. post feedback) was applied to ER, and RTs. In the case of neurophysiological measure $(\mathrm{O} 2 \mathrm{Hb})$ for the ANOVA two repeated factors were added to Cond, that is localization (Loc: DLPFC, DPMC, FEF, SFG) and lateralization (Lat: left vs. right) independent factor.

Successively, a similarity measure for continuous data (similarity measures for interval data, i.e. Pearson correlation as measure of distance between vectors, [40]) was applied to each dyad of subjects in pre- and post-feedback condition. By using this measure specific 
similarities between each dyad of subjects were monitored for cognitive (ER and RTs) and neurophysiological $(\mathrm{O} 2 \mathrm{Hb})$ dependent variables. Finally, to analyze the systematic effect of the independent within subjects factors condition (Cond) on the similarities data, repeated measure ANOVAs were applied to the similarity coefficients as dependent variables calculated for ER and RTs. In addition to Cond, in a successive ANOVA Loc and Lat factors were applied to the coefficients measured for $\mathrm{O} 2 \mathrm{Hb}$ dependent measure.

For all of the ANOVA tests, the degrees of freedom were corrected using GreenhouseGeisser epsilon where appropriate. Post-hoc comparisons (contrast analyses) were applied to the data. Bonferroni test was applied for multiple comparisons. In addition, the normality of the data distribution was preliminary tested (kurtosis and asymmetry tests). The normality assumption of the distribution was supported by these preliminary tests.

To exclude a possible learning effect, a preliminary analysis was applied, comparing separately the first set of four intervals (before feedback) and the second set four intervals (post feedback) for all the dependent measures (RTs, ERs, O2Hb). Since no significant differences among the four intervals respectively before and after the feedback were found, we did not include this factor in the successive analysis.

RTs and ERs

As shown by the ANOVA, no significant differences in ERs were found for Cond $(F[1$, $\left.23]=1.01, p \leq .001, \eta^{2}=.18\right)$. In contrast, for RTs, a significant effect was found for Cond $\left(F[1,23]=7.32, p \leq .001, \eta^{2}=.34\right)$, with increased RTs in post-feedback than pre-feedback (fig 3a-b) 


\section{fNIRS}

Repeated measure ANOVA showed significant effect for Cond $(F[1,24]=8.11, p \leq .001$, $\left.\eta^{2}=.36\right)$ and Cond $x$ Lat $x \operatorname{Loc}\left(F[1,84]=9.04, p \leq .001, \eta^{2}=.39\right)$. Indeed, a general increased activity was found in post-feedback condition than in pre-feedback. Secondly, as shown by contrast analyses applied to the simple effects, right DLPFC activity was increased than left DLPFC activity in post-feedback condition $\left(F[1,23]=8.90, p \leq .001, n^{2}=.35\right)$. In addition right DLPFC activity in post-feedback condition was increased than right DLPFC in pre-feedback condition $\left(F[1,23]=7.11, p \leq .001, \eta^{2}=.35\right)$. A significant effect was found also for SFG, with significant decreased responsiveness in post-feedback than pre-feedback condition, for both the left $\left(F[1,23]=7.90, p \leq .001, \eta^{2}=.33\right)$ and the right side $(F[1,23]=$ 7.88, $p \leq .001, \eta^{2}=.33$ ) (fig. 4a-c).

No other effect was statistically significant.

Correlational analysis

A series of correlation analysis was applied to cognitive performance (ERs; RTs) and $\mathrm{O} 2 \mathrm{Hb}$ modulation for each cortical area within the left and right hemisphere, distinctly for each condition (pre- and post-feedback). Pearson correlation coefficients were calculated between them: RTs revealed significant positive correlation with the right DLPFC in post-feedback condition $\left(\mathrm{r}^{2}=.523, p \leq .001\right)$ : the increased right DLPFC was positively correlated with the increased RTs values in post-feedback condition (fig 5a). In addition RTs were significantly correlated with SFG deactivation within the right and left hemisphere in post-feedback condition (respectively $\mathrm{r}^{2}=.499, p \leq .001 ; \mathrm{r}^{2}=-.521, p \leq .001$ ), with increased RTs in concomitance with reduced left and right SFG activity (fig 5b-c). 
Similarity measures

ER and RTs

The Pearson similarity coefficients were reported in the following figure (6a-b) for each couple of subjects in pre- and post-feedback. As indicated in figure 6a, for ER, nine couples showed significant coefficients for the pre-feedback condition, whereas three couples showed significant coefficients for the post-feedback condition. Figure $6 \mathrm{~b}$ indicates the coefficients for RTs measures. In this case, nine couples revealed significant joined RTs modulation for the pre-feedback condition, whereas four couples showed significant coefficients in postfeedback.

$\mathrm{O} 2 \mathrm{Hb}$

Significant Pearson coefficients were found in pre-feedback condition for nine couples in the right and eight in the left DLPFC, whereas six couples were matched in post-feedback in the right DLPFC and four couples in the left hemisphere of the DLPFC (fig 6c-d). significant coefficients were also found for SFG. Indeed two dyads were matched in pre-feedback condition in the right and two in the left hemisphere. In contrast, no significant coefficients were found for the post-feedback condition.

\section{ANOVA on similarity measures}

This level of analysis considered the Pearson coefficients derived for ER, RTs and O2Hb as dependent measure in the repeated measures ANOVAs. 
ERs and RTs coefficients

As shown by the ANOVA, significant differences in ER were found for Cond $(F[1,11]=$ $9.78, p \leq .001, \eta^{2}=.41$ ), with decreased Pearson coefficients values in post-feedback than pre-feedback condition. For RTs, a significant effect was found for Cond $(F[1,11]=8.15, p \leq$ $.001, \eta^{2}=.37$ ), with decreased Pearson values in post-feedback than pre-feedback.

\section{$\mathrm{O} 2 \mathrm{Hb}$ coefficients}

Repeated measure ANOVA showed significant effect for Cond $(F[1,11]=8.34, p \leq .001$, $\left.\eta^{2}=.38\right)$ and Cond $x$ Lat $x \operatorname{Loc}\left(F[1,33]=9.04, p \leq .001, \eta^{2}=.40\right)$. Indeed, decreased coefficients were found in post-feedback than pre-feedback condition. Secondly, about the interaction effect, during post-feedback the right hemisphere showed higher coefficient values compared to the left hemisphere in the DLPFC $\left(F[1,11]=7.56, p \leq .001, \eta^{2}=.35\right)$.

\section{Discussion}

The present research explored the effects of a negative social feedback on a joint-action, considering both the brain responsiveness and the cognitive performance. Specifically, the brain activation in dyads of subjects (inter-brain activity) was recorded during a cooperative task which was perceived as failing. Based on our results, the following points were observed. A first main effect was related to the systematic impact of the negative feedback on the cortical response, mainly for some specific frontal areas (DLPFC and SFG). Secondly, a specific lateralization effect was observable, in relation to the feedback. Indeed, the DLPFC showed a significant right lateralized activity in post-feedback than in pre-feedback condition. Thirdly a worse performance for RTs was revealed after the negatively reinforcing feedback. 
Finally, a gradual lower inter-brain synchronicity was found for the dyads during the task, after the negative feedback.

The first main effect was related to the increased DLPFC responsiveness after the subjects received their feedback. Indeed, we observed a general increased DLPFC activity in the case of a negative condition. This result may be coherent with the suggested hypothesis about the necessity to increase the cognitive effort and the processing load associated with the representation of a negative feedback with subsequent heightened cortical activity [23,27]. An unsuccessful strategy, although in a cooperative context, may require an increased demand of cognitive resources to update and modify the joint-action. As such, this condition may impose an increase in the cognitive load related to the necessity to recalibrate their own strategy, to implement a more efficient cognitive plan, and to include new behavioral directions. In addition, previous results revealed that prefrontal areas are prominent and relevant in social status regulation and joint actions $[11,41-43]$.

In the present research we observed a similar effect, with significant increased DLPFC activity in response to negatively reinforced joint action during the cognitive task. This prefrontal brain area was supposed to have an evolutionary relevance in social perception especially when the hierarchy across species and human social groups is crucial. Therefore it is plausible to suppose that this area reflects specialized mechanisms to perceive joint-actions. However, it should be noted that the effect we found was not generalized on the PFC, but in relation to the hemispheric lateralization, with a significant increased activation for the right DLPFC compared to the left one. This result may be explained based on our second hypothesis, which underlined the negative significance of an unsuccessful feedback or a competitive situation [5]. At this regard, we may consider the increased PFC right responsiveness as a possible marker reflecting the reduction of a self-perception of effectiveness and good performance. Indeed, as previously observed, the frontal cortical 
asymmetry in favor of the right hemisphere is associated with withdrawal motivation in opposition to approach motivation [44-49]. Therefore, we may explain these results also taking into account some previous results, on both cooperation and competition, where we found that the DLPFC was mainly activated within the left side in the case of positive cooperation [17] or within the right side in the case of competition $[6,18]$.

Therefore the present results seem to suggest that the negative cooperative condition is more similar to a competitive task and that this fact may be due to the increasing difficulty in creating a common mental strategy based on the increased work load; or secondarily due to the emotional negative condition that a negative feedback may create. However, the second explanation of the present result may bring the increased right responsiveness back to a significant prevalence of more negative and avoidance emotions toward the interlocutor, linked to the ineffective inter-action. In fact it was observed that the right hemisphere is supporting the aversive situations where the subjects have to regulate the conflictual and also divergent goals [5]. Therefore, a sort of a 'negative echo' may be intrinsically related to the failing, with a significant increasing of more withdrawal attitudes. Consequently, activity patterns in the frontal cortices can be regarded to be crucially involved in the processing of emotional conditions which characterize the negative context. Nevertheless, it has to be noted that few studies haye tried to connect the emotional effects of failing cooperation, taking into account the impact of the emotional behavior on the cortical system when it responds to specific social situations. Therefore future research should better explain the role of emotions and negative feedback to disambiguate their reciprocal relation.

An adjunctive interesting effect is related to the SFG decreased activity in response to negative feedback. Indeed, this area was found to be less involved after receiving the feedback. A possible interpretation of this result is related to the significance of this area for the cooperative situation. In fact it was found that the posterior regions of the right middle and 
superior frontal gyrus, in particular (BA8), are implicated during a cooperative interaction, suggesting that this area is involved in goal-oriented social interaction such as complex interactive movements and social decision-making [16]. The social relevance of the task and the cooperative condition may explain this result, while the concomitant decreased SFG response could be based on the 'weakened' cooperativity induced by a failing joint-behavior.

The present data were also supported by the behavioral results, that is a significant worse performance (increased RTs) after the negative feedback was observable. Although a cognitive effort due to the task after the feedback may not be excluded, we may suppose that the decreasing performance in post-feedback condition may be due to the negative selfperception and the representation of an inefficient interaction. Such results, in fact, are compatible with findings reported within the tradition of social psychology [50]. Starting from Bandura (see for example Bandura [51], other authors studied the relation between perceived self-efficacy and behavioral modifications. For example, Schunk conducted a series of studies with elementary school children who faced difficulties in Maths. The aim was to explore the effect of perceived self-efficacy as a function of different variables such as attributional feedback $[52,53]$, reward contingencies [53,54], but, more importantly, social comparison [55]. Thus, in many studies, he reported that the degree of self-efficacy was accompanied by a modulation in performance and behavior.

Moreover, a simple working load effect is not compatible with our preliminary analysis which further tested the absence of significant effects within each of the four intervals (no performance decreasing based on the comparison between the four time intervals for pre- nor for post-feedback condition).

It should also be noted that, based on correlational measures, the cortical and behavioral data showed to be matched, with a similar trend of increased values for both cognitive behavior (higher RTs) and cortical activity (increased DLPFC and reduced SFG 
responsiveness), which underlined the main effect of the (artificially) induced negative social reinforce on the inter-subjective joint performance.

Finally, about the inter-brain response, we observed a consistent and relevant decreased shared activity for the dyads, mainly in concomitance to the post-feedback condition, taking into account both cognitive and neuropsychological levels. That is, this "divergent" interbrain activity emerged after a negative feedback condition. Therefore it could be suggested that, due to the negative joined performance, the self-perceived low efficacy produced a sort of "disconnection" between the two brains, orienting the subjects on the divergent direction. It should be considered that, although the synergic strategy tended to become less consistent, the right hemisphere maintains a higher synchronicity than the left, as shown by the similarity measures. This fact may be related to the negative emotional effect we supposed to be present after the negative feedback on participants' behavior. In other words, the negative value of the post-feedback condition may have ingenerated a joined response for the dyad, with similar negative emotional response within the right hemisphere which is generally deputed to control the negative affect.

More generally, the current research provides initial evidence for the hypothesis of a significant inter-brain effect during a cooperation which fails and lays a foundation for future research examining the extent to which the negative condition is selectively related to a worse cognitive joint performance and reduced inter-brain synergy for the two inter-agents.

To conclude, negative feedback ingenerates some behavioral and brain responses similar to a sort of 'competitive context', or a context which is affected by negative emotions. Some specific areas (mainly the right DLPFC) appeared to be highly implicated as a marker of this social negative effect, where subjects had to recalibrate their strategy and to manage negative feeling linked to the inefficient performance. The social relevance of this negative feedback (SFG) and the emotional impact of this unpleasant condition (right DLPFC) could make the 
cooperation less 'cooperative' and the strategic plan less effective (low inter-brain synergy) and more comparable to a 'competitive' condition.

\section{Acknowledgements}

None

\section{Declaration of interest statement}

The authors report no conflicts of interest

\section{Geolocation}

Milan 


\section{References}

1. Balconi M, Bortolotti A. Resonance mechanism in empathic behavior. BEES, BIS/BAS and psychophysiological contribution. Physiol. Behav. [Internet]. Elsevier Inc.; 2012;105:298-304. Available from: http://dx.doi.org/10.1016/j.physbeh.2011.08.002

2. Liu T, Saito H, Oi M. Role of the right inferior frontal gyrus in turn-based cooperation and competition: a near-infrared spectroscopy study. Brain Cogn. [Internet]. Elsevier Inc.; 2015;99:17-23. Available from: http://dx.doi.org/10.1016/j.bandc.2015.07.001

3. Baker JM, Liu N, Cui X, Vrticka P, Saggar M, Hosseini SMH, et al. Sex differences in neural and behavioral signatures of cooperation revealed by fNIRS hyperscanning. Sci. Rep. 2016;6:26492.

4. Vanutelli ME, Nandrino J-L, Balconi M. The boundaries of cooperation: sharing and coupling from ethology to neuroscience. Neuropsychol. Trends [Internet]. 2016;19:83-104. Available from: http://www.ledonline.it/NeuropsychologicalTrends/19-2016.html

5. Balconi M, Falbo L, Conte VA. BIS and BAS correlates with psychophysiological and cortical response systems during aversive and appetitive emotional stimuli processing. Motiv. Emot. 2012;36:218-31.

6. Baleoni M, Pagani S. Social hierarchies and emotions: cortical prefrontal activity, facial feedback (EMG), and cognitive performance in a dynamic interaction. Soc. Neurosci. [Internet]. 2015;10:166-78. Available from: http://www.ncbi.nlm.nih.gov/pubmed/25372808

7. Chung D, Yun K, Jeong J. Decoding covert motivations of free riding and cooperation from multi-feature pattern analysis of EEG signals. Soc. Cogn. Affect. Neurosci. 2015;10:1210-8. 
8. Cui X, Bryant DM, Reiss AL. NIRS-based hyperscanning reveals increased interpersonal coherence in superior frontal cortex during cooperation. Neuroimage [Internet]. 2012;59:2430-7. Available from: http://dx.doi.org/10.1016/j.neuroimage.2011.09.003

9. Funane T, Kiguchi M, Atsumori H, Sato H, Kubota K, Koizumi H. Synchronous activity of two people's prefrontal cortices during a cooperative task measured by simultaneous nearinfrared spectroscopy. J. Biomed. Opt. 2011;16:77011.

10. Goldman M, Stockbauer JW, McAuliffe TG. Intergroup and intragroup competition and cooperation. J. Exp. Soc. Psychol. 1977;113:81-8.

11. Suzuki S, Niki K, Fujisaki S, Akiyama E. Neural basis of conditional cooperation. Soc Cogn Affect Neurosci [Internet] 2011;6:338-47. Available from: http://www.ncbi.nlm.nih.gov/pmc/articles/PMC3110432/pdf/nsq042.pdf

12. Levitan R, Hasey G, Sloman L. Major depression and the involuntary defeat strategy: biological correlates. In: Gilbert P, Sloman L, editors. Subordination defeat an Evolotuinary approach to mood Disorders and Their Therapy. Manhaw: Lawrence Erlbaum Associates; 2000. p. 95-120.

13. Balconi M, Pagani S. Personality correlates (BAS-BIS), self-perception of social ranking, and cortical (alpha frequency band) modulation in peer-group comparison. Physiol. Behav. $2014 ; 133 C: 207-15$

14. Chiao JY, Adams RBJ, Tse PU, Lowenthal L, Richeson JA, Ambady N. Knowing who's boss: fMRI and ERP investigations of social dominance perception. Gr. Process. Intergr. Relations. 2009;11:201-14.

15. Marsh AA, Blair KS, Jones MM, Soliman N, Blair RJR. Dominance and submission: the ventrolateral prefrontal cortex and responses to status cues. 2009;21:713-24. 
16. Liu N, Mok C, Witt EE, Pradhan AH, Chen JE, Reiss AL. NIRS-based hyperscanning reveals inter-brain neural synchronization during cooperative Jenga game with face-to-face communication. Front. Hum. Neurosci. [Internet]. Frontiers Media S.A.; 2016;10:82. Available from: http://www.ncbi.nlm.nih.gov/pmc/articles/PMC4782164/

17. Balconi M, Vanutelli ME. Interbrains cooperation: Hyperscanning and self-perception in joint actions. J. Clin. Exp. Neuropsychol. Routledge; 2017;13:1-14.

18. Balconi M, Vanutelli ME. Competition in the brain. The contribution of EEG and fNIRS modulation and personality effects in social ranking. Front. Psychol. [Internet]. 2016;7:1587. Available from: http://www.ncbi.nlm.nih.gov/pmc/articles/PMC5062540/

19. Monterosso J, Ainslie G, Pamela Toppi Mullen PA-C, Gault B. The fragility of cooperation: A false feedback study of a sequential iterated prisoner's dilemma. J. Econ. Psychol. [Internet]. 2002;23:437-48. Available from: file://www.sciencedirect.com/science/article/pii/S0167487002000958

20. Holper L, Scholkmann F, Wolf M. Between-brain connectivity during imitation measured by fNIRS. Neuroimage [Internet]. 2012;63:212-22. Available from: http://dx.doi.org/10.1016/j.neuroimage.2012.06.028

21. Sebanz N, Knoblich G, Prinz W. Representing others' actions: just like one's own? Cognition. 2003,88:B11-21.

22. Knoblich G, Jordan JS. Action coordination in groups and individuals: learning anticipatory control. J. Exp. Psychol. Learn. Mem. Cogn. 2003;29:1006-1016.

23. Gallagher HL, Frith CD. Functional imaging of "theory of mind." Trends Cogn. Sci. $2003 ; 7: 77-83$. 
24. Leslie A. Pretense and representation: the origins of "“Theory of Mind." Psychol. Rev. 1987;94:412-26.

25. Nigg JT. Is ADHD a disinhibitory disorder? Psychol. Bull. 2001;127:571-98.

26. Humphrey NK. The social function of intellect. In: Byrne RW, Whiten A, editors. Machiavellian Intell. Soc. Expert. Evol. intellect monkeys, apes, humans. Oxford: Clarendon; 1988. p. 13-26.

27. Decety J, Jackson PL, Sommerville JA, Chaminade T, Meltzoff AN. The neural bases of cooperation and competition: An fMRI investigation. Neuroimage. 2004,23:744-51.

28. Balconi M, Canavesio Y. High-frequency rTMS on DLPFC increases prosocial attitude in case of decision to support people. Soc. Neurosci. [Internet]. 2014;9:82-93. Available from: http://www.ncbi.nlm.nih.gov/pubmed/24279315

29. Balconi M, Canavesio Y. Prosocial attitudes and empathic behavior in emotional positive versus negative situations: brain response (ERPs) and source localization (LORETA) analysis. Cogn. Process. 2013;14:63-72.

30. Balconi M, Bortolotti A, Gonzaga L. Emotional face recognition, EMG response, and medial prefrontal activity in empathic behaviour. Neurosci. Res. [Internet]. Elsevier Ireland Ltd and Japan Neuroscience Society; 2011;71:251-9. Available from: http://dx.doi.org/10.1016/j.neures.2011.07.1833

31. Elwell CE, Owen-Reece H, Cope M, Wyatt JS, Edwards AD, Delpy DT, et al. Measurement of adult cerebral haemodynamics using near infrared spectroscopy. Acta Neurochir. Suppl. (Wien). 1993;59:74-80.

32. Tuscan LA, Herbert JD, Forman EM, Juarascio AS, Izzetoglu M, Schultheis M. Exploring 
frontal asymmetry using functional near-infrared spectroscopy: A preliminary study of the effects of social anxiety during interaction and performance tasks. Brain Imaging Behav. 2013;7:140-53.

33. Beck AT, Steer RA, Brown GK. Manual for the Beck Depression Inventory - II. San Antonio: Psychological Corporation; 1996.

34. Spielberger CD, Gorsuch RL, Lushene RE, Vagg PR, Jacobs GA. STAI Manual for the State-Trait Anxiety Inventory. Palo Alto: Consulting Psychologists Press; 1970.

35. Koessler L, Maillard, L.Benhadid A, Vignal JP, Felblinger J, Vespignani H, Braun M. Automated cortical projection of EEG sensors: Anatomical correlation via the international 10-10 system. Neuroimage [Internet]. 2009;46:64-72. Available from: http://dx.doi.org/10.1016/j.neuroimage.2009.02.006

36. Winer BJ, Brown DR, Michels KM. Statistical Principles in Experimental Design. 3rd ed. New York: McGraw-Hill; 1991.

37. Schroeter ML, Zysset S, Kruggel F, Von Cramon DY. Age dependency of the hemodynamic response as measured by functional near-infrared spectroscopy. Neuroimage. $2003 ; 19: 555-64$.

38. Matsuda G, Hiraki K. Sustained decrease in oxygenated hemoglobin during video games in the dorsal prefrontal cortex: A NIRS study of children. Neuroimage. 2006;29:706-11.

39. Shimada S, Hiraki K. Infant's brain responses to live and televised action. Neuroimage. 2006;32:930-9.

40. Ashby FG, Perrin NA. Toward a unified theory of similarity and recognition. Psychol. Rev. 1988;95:124-50. 
41. De Vico Fallani F, Nicosia V, Sinatra R, Astolfi L, Cincotti F, Mattia D, et al. Defecting or not defecting: how to "read" human behavior during cooperative games by EEG measurements. PLoS One [Internet]. 2010;5:e14187. Available from: http://dx.plos.org/10.1371/journal.pone.0014187

42. Haruno M, Kawato M. Activity in the superior temporal sulcus highlights learning competence in an interaction game. J. Neurosci. 2009;29:4542-7.

43. Karafin MS, Tranel D, Adolphs R. Dominance attributions following damage to the ventromedial prefrontal cortex. J. Cogn. Neurosci. 2004;16:1796-804

44. Balconi M, Mazza G. Lateralisation effect in comprehension of emotional facial expression: a comparison between EEG alpha band power and behavioural inhibition (BIS) and activation (BAS) systems. Laterality. 2010;15:361-84.

45. Davidson RJ. Cerebral asymmetry and emotion: conceptual and methodological conundrums. Cogn. Emot. 1993;7:115-38.

46. Harmon-Jones E, Gable PA, Peterson CK. The role of asymmetric frontal cortical activity in emotion-related phenomena: a review and update. Biol. Psychol. [Internet]. Elsevier B.V.; 2010;84:451-62. Ayailable from: http://dx.doi.org/10.1016/j.biopsycho.2009.08.010

47. Jackson DC, Mueller CJ, Dolski I, Dalton KM, Nitschke JB, Urry HL, et al. Now you feel it, now you don't: frontal brain electrical assymetry and individual differences in emotion regulation. Psychol. Sci. 2003;14:612-7.

48. Koslow K, Mendes WB, Pajtas PE, Pizzagalli DA. Greater left resting intracortical activity as a buffer to social threat. Psychol. Sci. 2013;22:641-9.

49. Urry HL, Nitschke JB, Dolski I, Jackson DC, Dalton KM, Mueller CJ, et al. Making a life 
worth living. Neural correlates of well-being. Psychol. Sci. 2004;15:367-72.

50. Bouffard-Bouchard T. Influence of self-efficacy on performance in a cognitive task. J. Soc. Psychol. [Internet]. Routledge; 1990;130:353-63. Available from: http://dx.doi.org/10.1080/00224545.1990.9924591

51. Bandura A. Self-efficacy: Toward a unifying theory of behavioral change. Psychol. Rev. 1977;84:191.

52. Schunk DH. Modeling and attributional effects on children's achievement: A self-efficacy analysis. J. Educ. Psychol. 1981;73:93-105.

53. Schunk DH. Reward contingencies and the development of children's skills and selfefficacy. J. Educ. Psychol. 1983;75:511-8.

54. Schunk DH. Self-efficacy perspective on achievement behavior. Educ. Psychol. 1984;19:48-58.

55. Schunk DH. Developing children's self-efficacy and skills: The roles of social comparative information and goal setting. Contemp. Educ. Psychol. 1983;8:76-86. 


\section{Figure Caption}

Fig 1 Experimental procedure which represents the setting, the attentional task and fNIRS recording

Fig 2 The location of NIRS channels. The emitters (red) were placed on positions FC3-FC4 and F1-F2, while detectors (fuchsia) were placed on FC1-FC2 and F3-F4. Resulting channels (yellow) were as follows: Ch 1 and Ch 3 correspond to the left and right DLPFC. Ch 2 and Ch 4 correspond to the left and right PMC. Ch 5 and $\mathrm{Ch} 7$ correspond to the left and right FEF. Ch 6 and Ch 8 correspond to the left and right SFG

Fig 3 (a) ERs modulation with no significant effects. (b) RTs modulation as a function of preand post-feedback conditions. The post-feedback condition was characterized by longer RTs

Fig $4 \mathrm{O} 2 \mathrm{Hb}$ variations (D values). The post-feedback condition was characterized by (a) increased D values over the right DLPFC and (b) general decreased D values for both left and right side over the SFG in O2Hb levels; (c) the cortical maps related to DLPFC and SFG as a function of pre- and post-feedback

Fig 5 Correlational values (Pearson coefficient) for: (a) right DLPFC, increased values significantly correlated with increased RTs values; (b) left and (c) right SFG, decreased values significantly correlated with increased RTs

Fig 6 Similarity measures for: (a) ERs; (b) RTs; (c) O2Hb for left DLPFC; (d) O2Hb for right DLPFC 


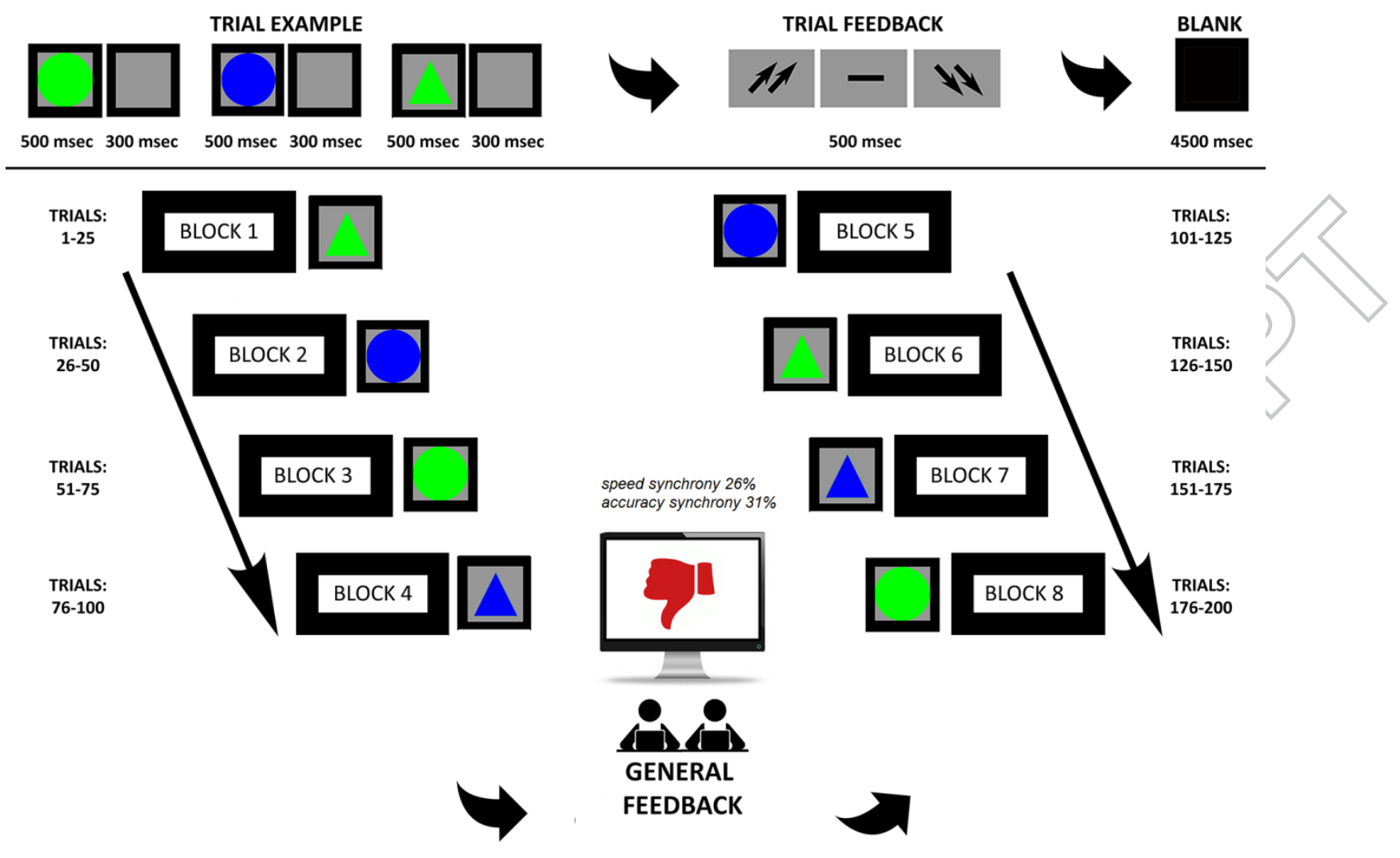

Fig 1 Experimental procedure which represents the setting, the attentional task and fNIRS recording 


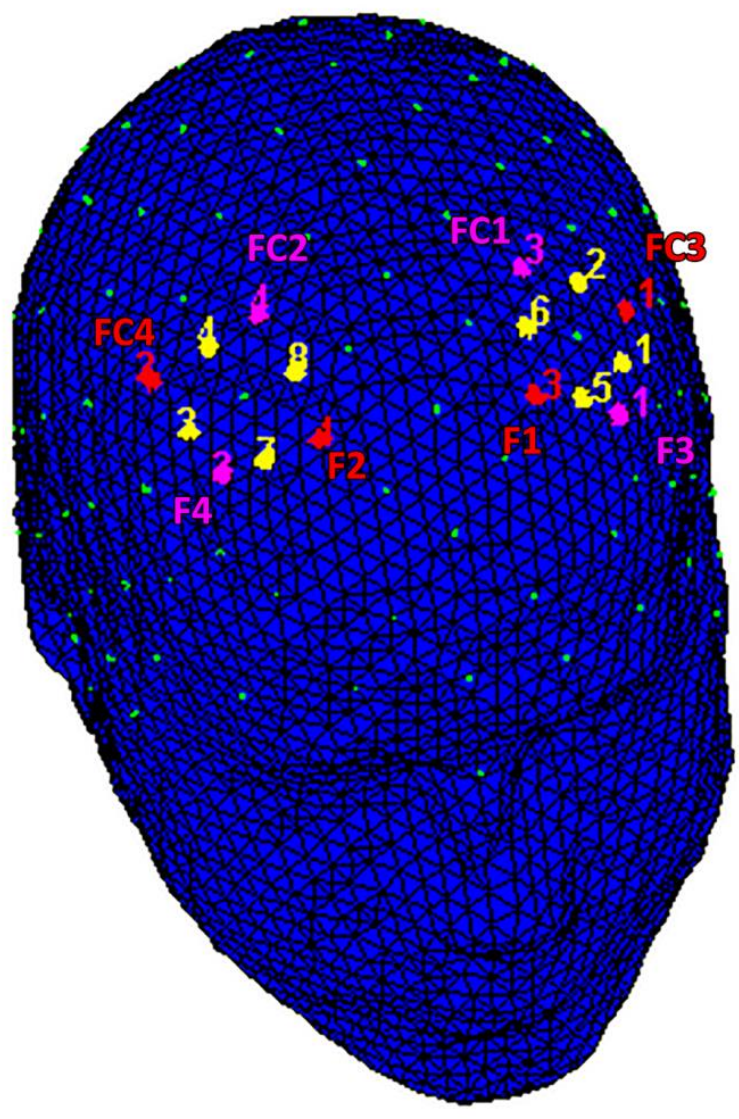

Fig 2 The location of NIRS channels. The emitters (red) were placed on positions FC3-FC4 and F1-F2, while detectors (fuchsia) were placed on FC1-FC2 and F3-F4. Resulting channels (yellow) were as follows: $\mathrm{Ch} 1$ and $\mathrm{Ch} 3$ correspond to the left and right DLPFC. Ch 2 and Ch 4 correspond to the left and right PMC. Ch 5 and $\mathrm{Ch} 7$ correspond to the left and right FEF. Ch 6 and Ch 8 correspond to the left and right SFG 


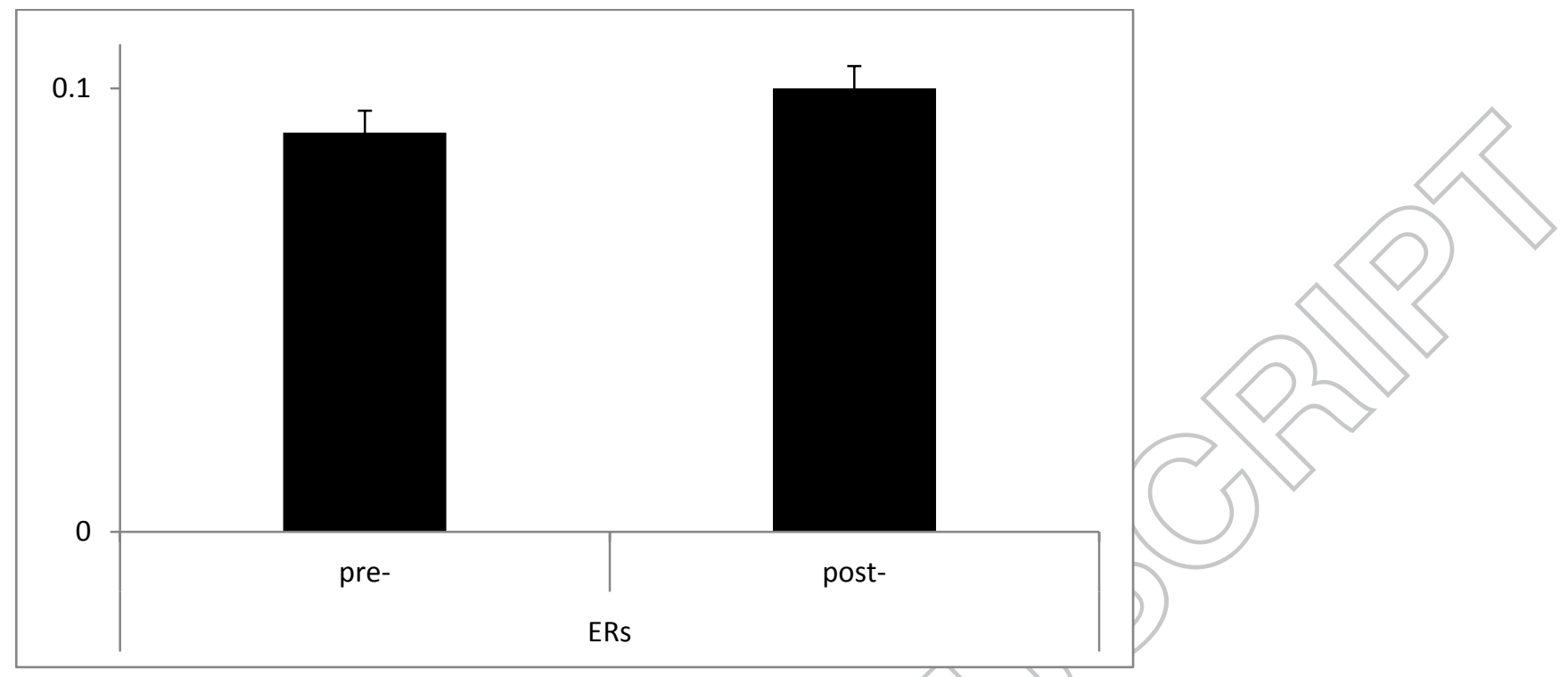

Fig $3 a$

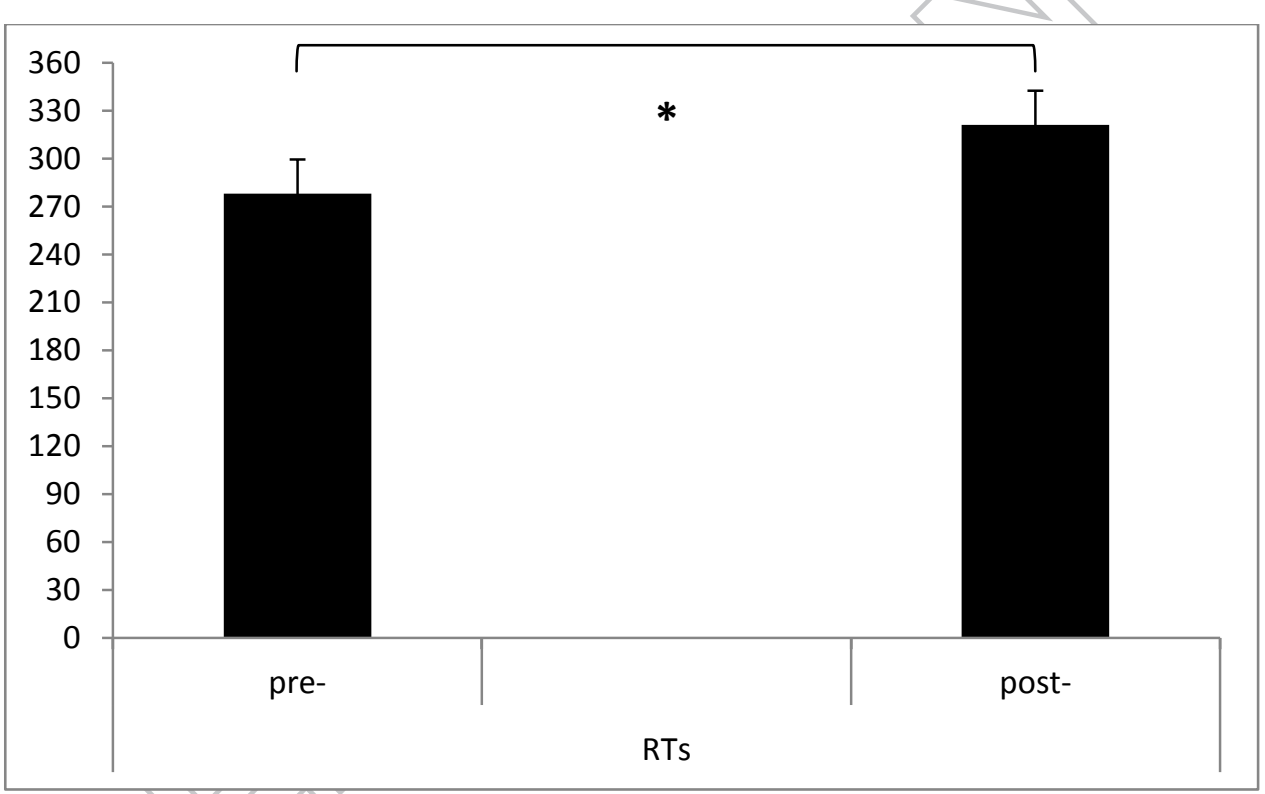

Fig $3 b$

Fig 3 (a) ERs modulation with no significant effects. (b) RTs modulation as a function of preand post-feedback conditions. The post-feedback condition was characterized by longer RTs 


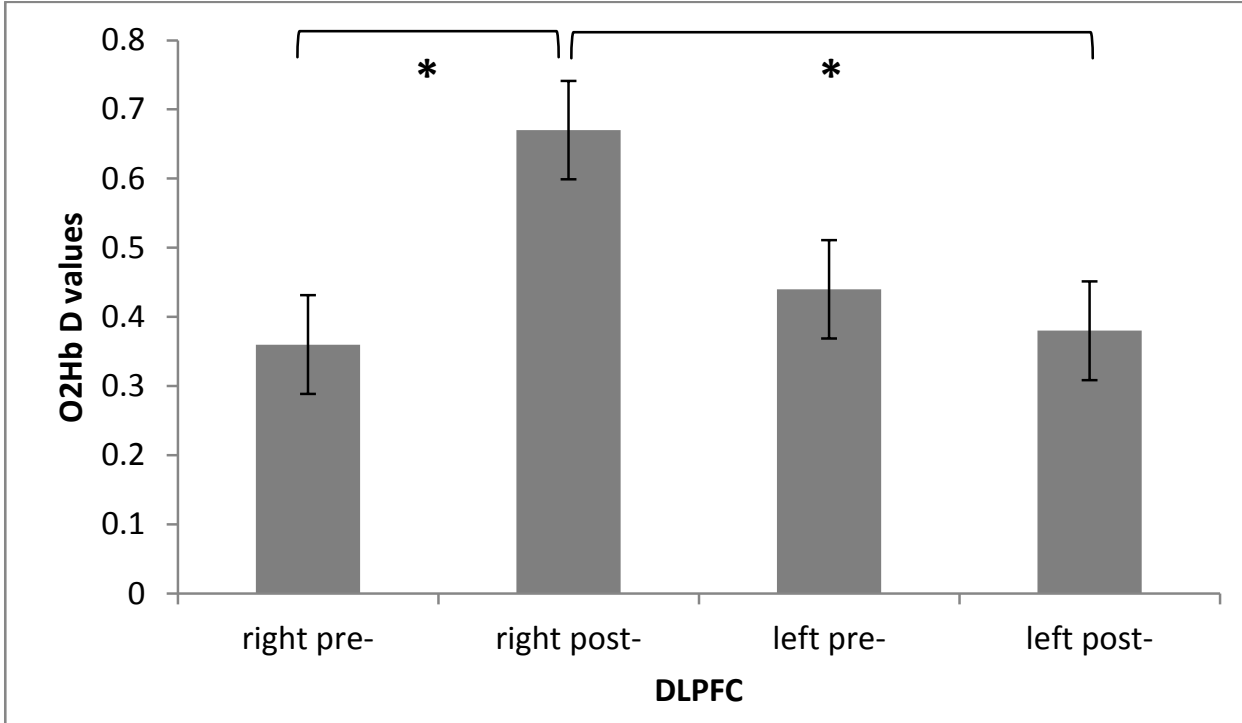

Fig $4 a$

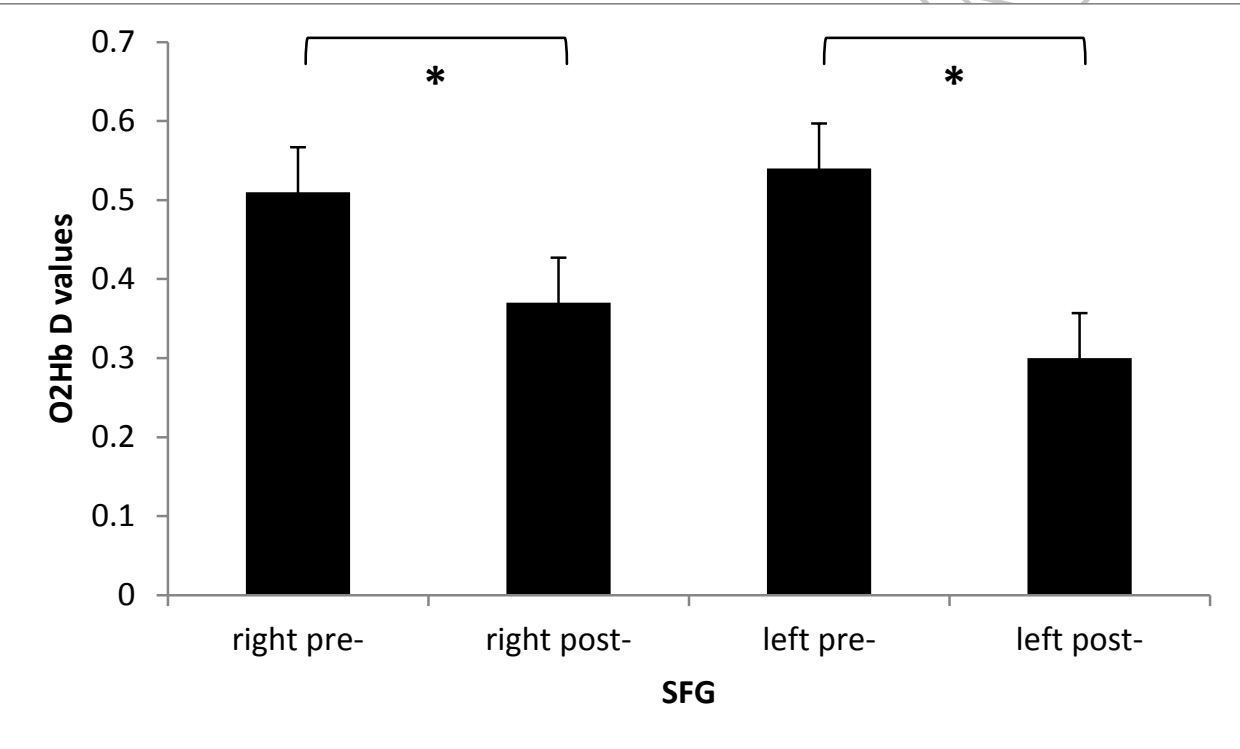

Fig $4 \mathrm{~b}$

Fig $4 \mathrm{O} 2 \mathrm{Hb}$ variations (D values). The post-feedback condition was characterized by (a) increased D values over the right DLPFC and (b) general decreased D values for both left and right side over the SFG in O2Hb levels; (c) the cortical maps related to DLPFC and SFG as a function of pre- and post-feedback 


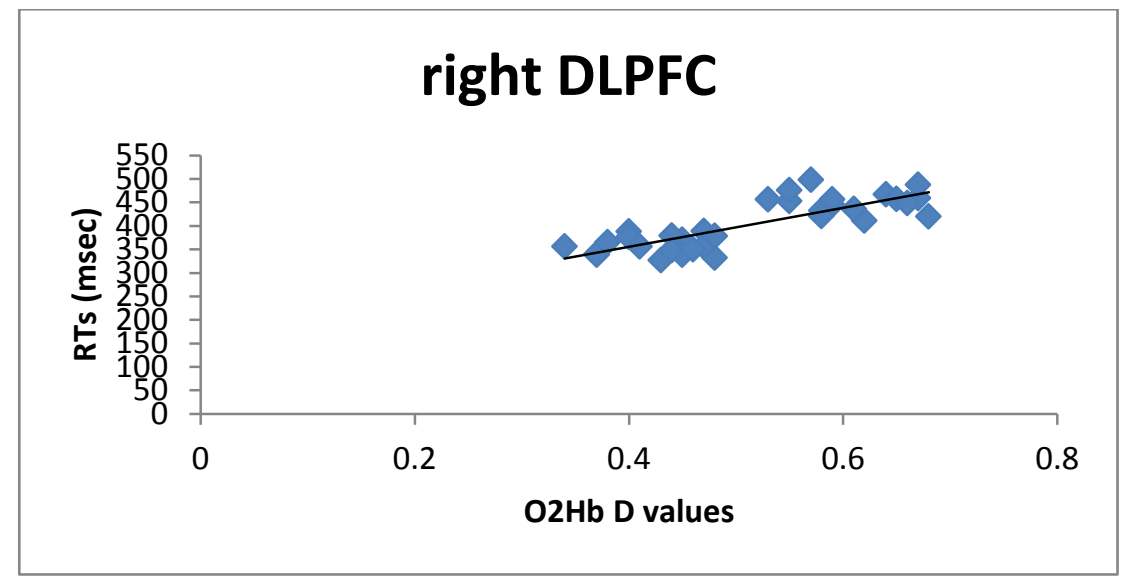

Fig $5 a$

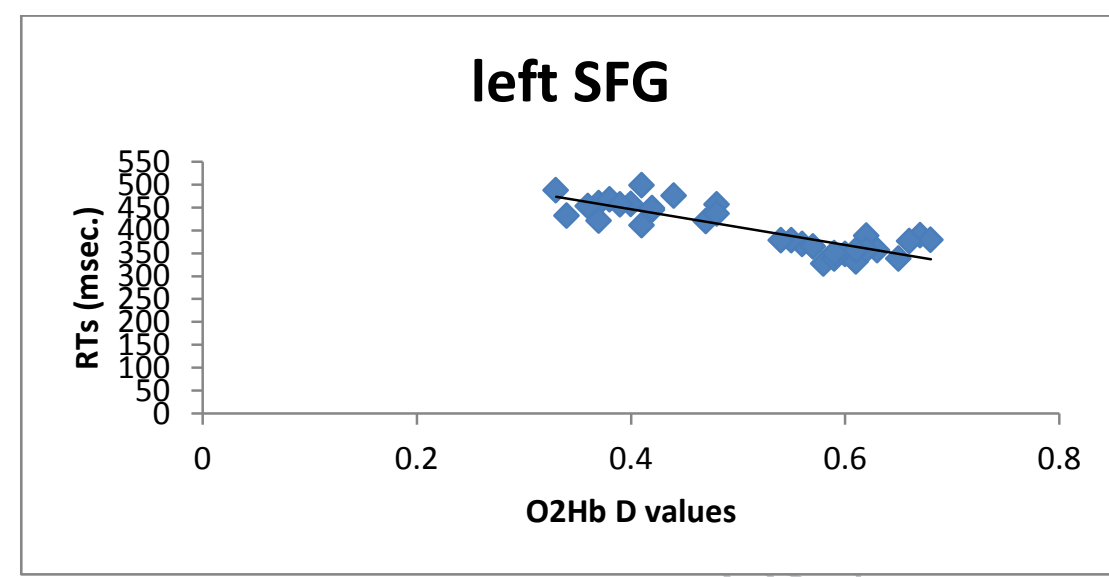

Fig $5 b$

right SFG

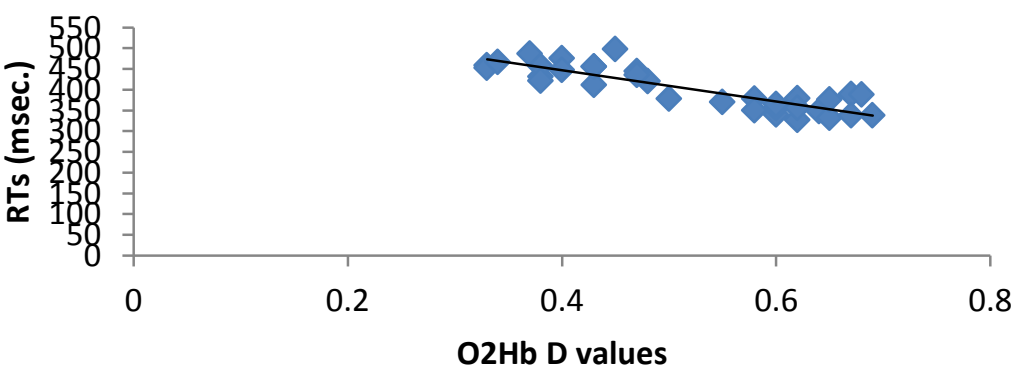

Fig 5c

Fig 5 Correlational values (Pearson coefficient) for: (a) right DLPFC, increased values significantly correlated with increased RTs values; (b) left and (c) right SFG, decreased values significantly correlated with increased RTs 


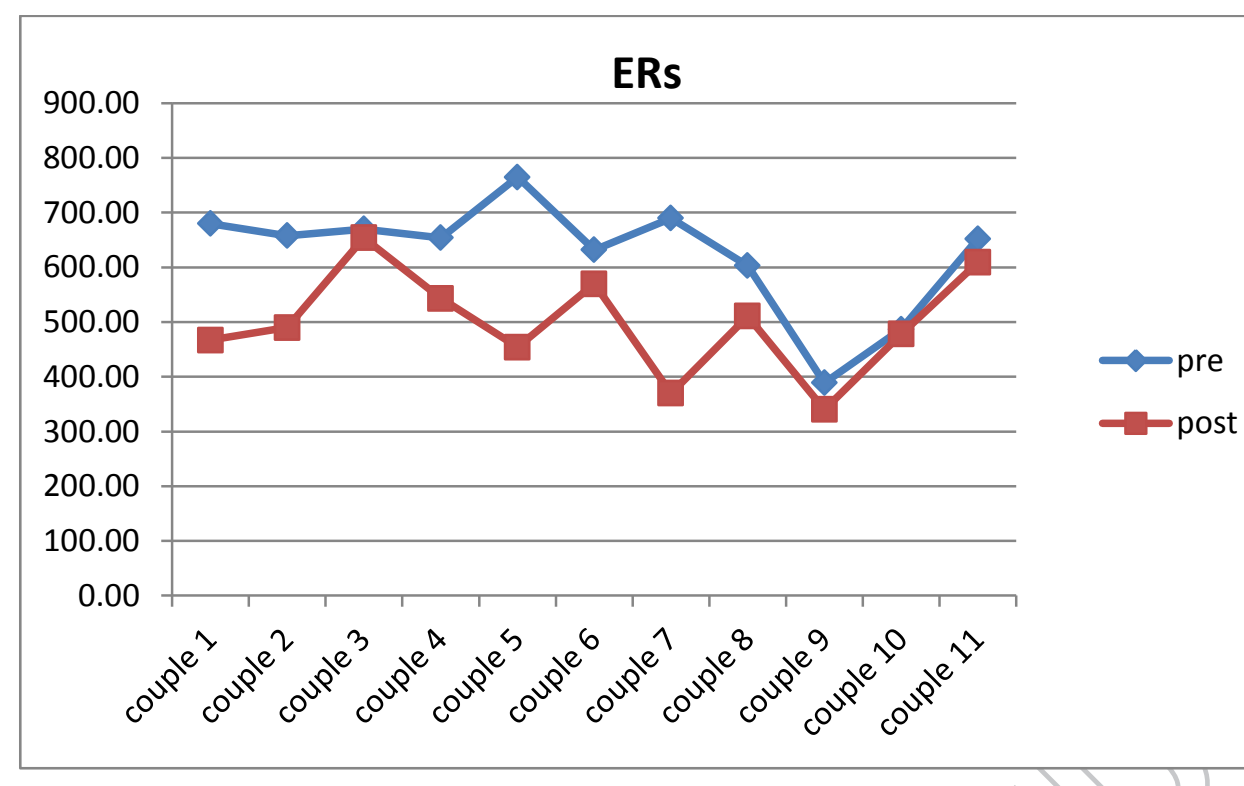

\begin{tabular}{|ccc|}
\hline & pre & post \\
couple 1 & $680^{*}$ & 467 \\
couple 2 & $658^{*}$ & 490 \\
couple 3 & $67 *^{*}$ & $654^{*}$ \\
couple 4 & $654^{*}$ & 543 \\
couple 5 & $765^{*}$ & 454 \\
couple 6 & $632^{*}$ & $570^{*}$ \\
couple 7 & $690^{*}$ & 370 \\
couple 8 & $603^{*}$ & 511 \\
couple 9 & 389 & 340 \\
couple 10 & 487 & 478 \\
couple 11 & $652^{*}$ & $609 *$ \\
\hline
\end{tabular}

Fig 6a 


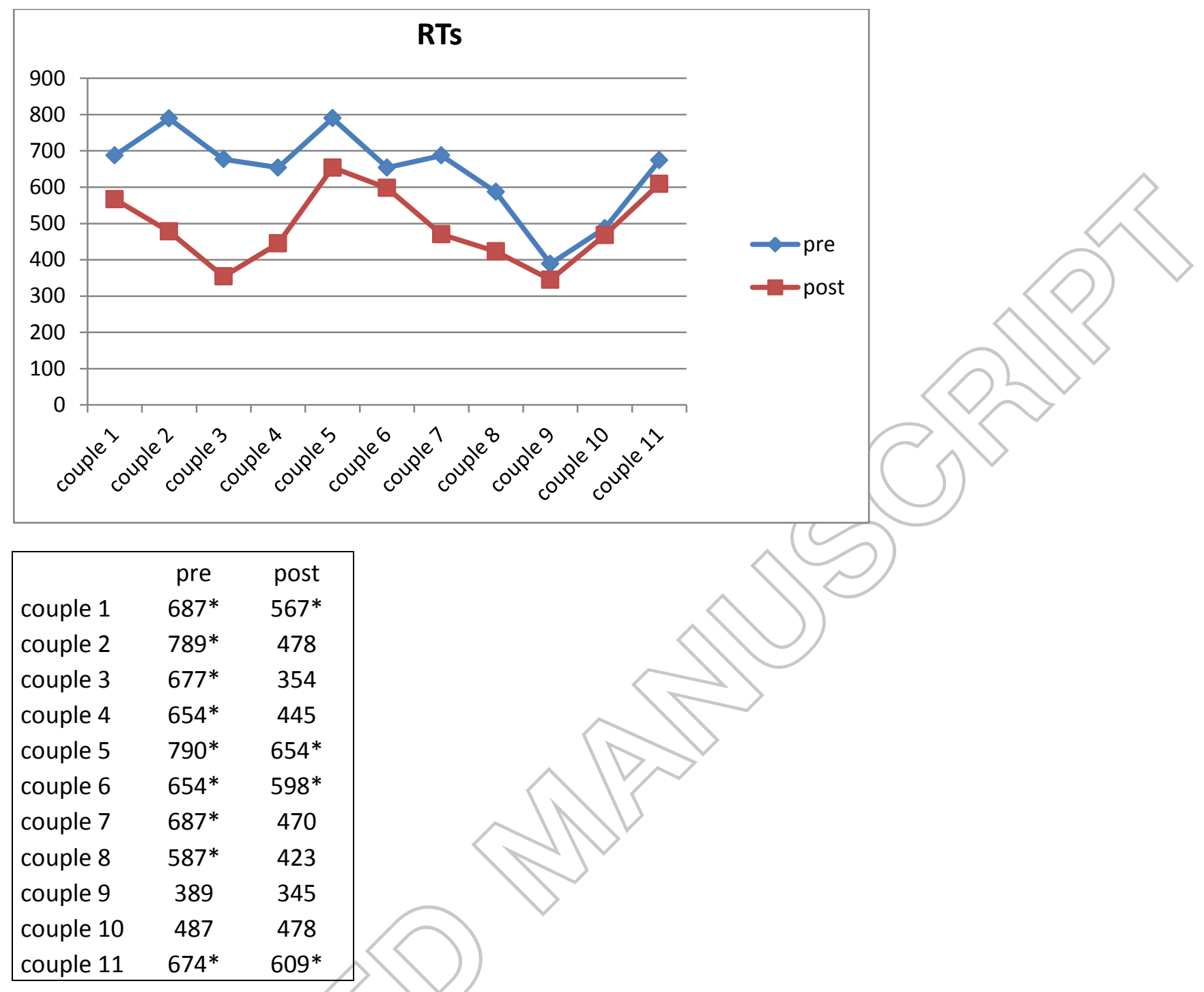

Fig $6 b$ 


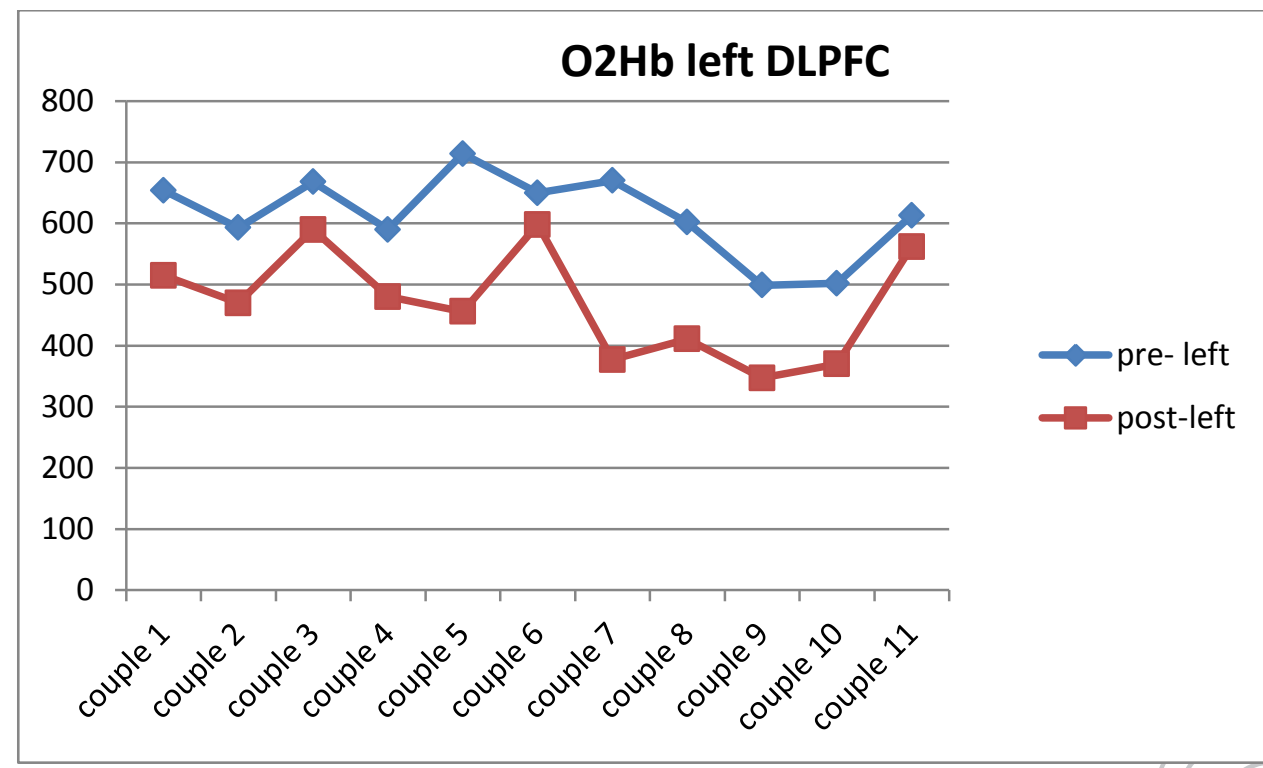

\begin{tabular}{|lcc|}
\hline & pre-left & post-left \\
couple 1 & $654^{*}$ & $515^{*}$ \\
couple 2 & 593 & 470 \\
couple 3 & $668^{*}$ & $590^{*}$ \\
couple 4 & $590^{*}$ & 480 \\
couple 5 & $714^{*}$ & 456 \\
couple 6 & $650^{*}$ & $598^{*}$ \\
couple 7 & $670^{*}$ & 377 \\
couple 8 & $602^{*}$ & 411 \\
couple 9 & 499 & 347 \\
couple 10 & $502^{*}$ & 370 \\
couple 11 & $613^{*}$ & $562^{*}$ \\
\hline
\end{tabular}

Fig 6c 


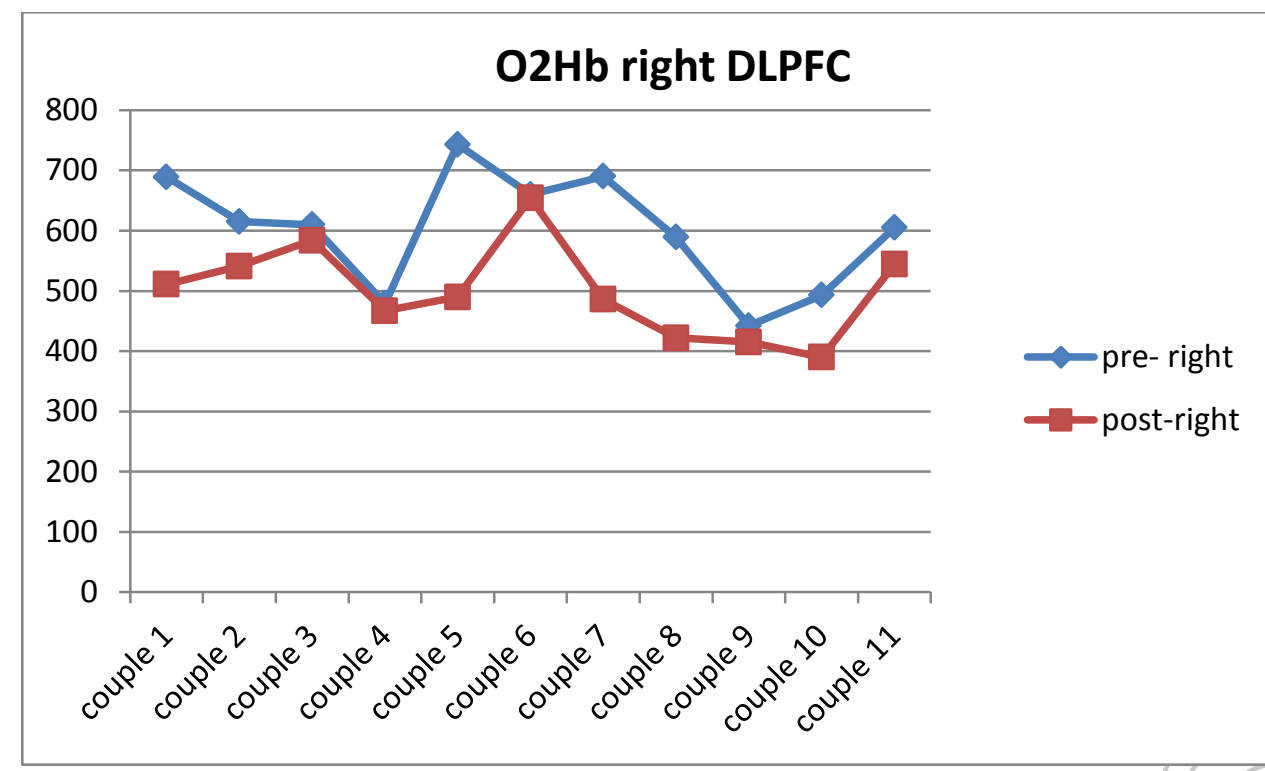

\begin{tabular}{|lcc|}
\hline & pre-right & post-right \\
couple 1 & $689^{*}$ & $511^{*}$ \\
couple 2 & $615^{*}$ & $541^{*}$ \\
couple 3 & $610^{*}$ & $584^{*}$ \\
couple 4 & 479 & 467 \\
couple 5 & $743^{*}$ & $490^{*}$ \\
couple 6 & $660^{*}$ & $654^{*}$ \\
couple 7 & $690^{*}$ & 486 \\
couple 8 & $589^{*}$ & 422 \\
couple 9 & 442 & 415 \\
couple 10 & 493 & 390 \\
couple 11 & $605^{*}$ & $544^{*}$ \\
\hline
\end{tabular}

Fig 6d

Fig 6 Similarity measures for: (a) ERs; (b) RTs; (c) O2Hb for left DLPFC; (d) O2Hb for right DLPFC 